\title{
Pore space relevant for gas permeability in Opalinus clay: Statistical analysis of homogeneity, percolation, and representative volume element
}

\author{
Lukas M. Keller, ${ }^{1}$ Lorenz Holzer, ${ }^{1}$ Philipp Schuetz, ${ }^{2}$ and Philippe Gasser ${ }^{3}$ \\ Received 28 January 2013; revised 18 May 2013; accepted 23 May 2013; published 18 June 2013.
}

[1] Local porosity theory in combination with percolation theory was applied to shale microstructures that were reconstructed on the basis of focused ion beam nanotomography and scanning transmission electron microscopy. This allowed characterizing pore microstructures in Opalinus clay with length scales on the order of tens of microns. In a sample from the sandy facies (with low clay content), the fraction of "larger" pores $\phi($ radii $\sim>15 \mathrm{~nm})=0.076$ is substantially higher than that in the shaley facies (with a higher clay content $)$, where $\phi($ radii $>15 \mathrm{~nm})=0.015$. The resolved porosity possesses a certain degree of homogeneity, and the representative volume element (RVE) of porosity can be determined in terms of a given relative error on porosity. For example, if we accept a relative error of $10 \%$, the RVE is on the scale of a few hundreds of microns. Both pore microstructures from sandy and shaley facies show anisotropic characteristics with respect to connectivity and percolation threshold. Using finite scaling, we found percolation thresholds with critical porosities $\phi_{c, b}=0.04-0.12$ parallel to bedding and $\phi_{c \text {,perp }}=0.11-0.19$ perpendicular to bedding. The resolved porosity of the sandy facies (low clay content) is close to the percolation threshold, whereas the porosity of the shaley facies (high clay content) is below the percolation threshold. The porosity in carbonate layers is around $\phi=0.027$, and the pore size is substantially larger when compared to the pores in the clay matrix. In the analyzed sample, pores in carbonate layers are poorly connected.

Citation: Keller, L. M., L. Holzer, P. Schuetz, and P. Gasser (2013), Pore space relevant for gas permeability in Opalinus clay: Statistical analysis of homogeneity, percolation and representative volume element, J. Geophys. Res. Solid Earth, 118, 2799-2812, doi:10.1002/jgrb.50228.

\section{Introduction}

[2] In the present investigation, the pore structure of clay rocks and its influence on gas permeability is investigated. This topic is of importance, e.g., for the exploitation of natural gas reservoirs from so-called gas shales. In addition, shaley rocks such as the Opalinus clay are also considered as potential host rocks for disposals of radioactive waste [Andra, 2005; Nagra, 2002, 2004]. Thereby, gas permeability is of interest, for example, in the context of gas pressure from corrosion and for the associated pressure release [e.g., Horseman et al., 1996].

[3] In general, shales are often thought to be relatively impermeable to gas, because porosity that is relevant for transport (i.e., pores with radii $>\sim 10 \mathrm{~nm}$ ) is low in these rocks. This

\footnotetext{
${ }^{1}$ Zürich University of Applied Sciences, Institute of Computational Physics, Winterthur, Switzerland.

${ }^{2}$ Materials Science and Technology, Laboratory for Electronics/ Metrology/Reliability, Dübendorf, Switzerland.

${ }^{3}$ Centre for Imaging Science and Technology, Swiss Federal Institute of Technology, Zürich, Switzerland.

Corresponding author: L. Keller, Zürich University of Applied Sciences, CH-8400 Winterthur, Switzerland. (kelu@zhaw.ch)

(C)2013. American Geophysical Union. All Rights Reserved. 2169-9313/13/10.1002/jgrb.50228
}

may mean that the volume fraction of larger pores is below the critical value for percolation. The restriction of gas transport to larger pores (i.e., pores with radii $>\sim 10 \mathrm{~nm}$ ) is made because gas flow along smaller pores is hampered due to capillary forces [Clayton and Hay, 1994; Marschall et al., 2005].

[4] However, the percolation threshold related to gas transport in shales is not known in detail. So far, microstructural studies on shales focused on clay matrix connectivity [Cosenza et al., 2012; Robinet et al., 2012], whereas studies on pore connectivity are largely absent. The main challenges of such studies lie in the preparation of the samples so that they can be used for focused ion beam (FIB), which occurs under high vacuum. The above pore sizes can be resolved by FIB nanotomography (FIB-nt), which reaches resolutions (i.e., voxel sizes) down to about $5 \mathrm{~nm}$. Therefore, the method is very useful to examine potential pathways for gas transport. Here we used cryosample preparation in combination with FIB in order to obtain a 3-D reconstruction of the pore space, on which base the percolation threshold can be calculated. In doing so, we used a two-step approach. In the first step, we applied the local porosity theory (LPT) [Hilfer, 1991; Biswal et al., 1998; Hu and Stroeven, 2005] for the analysis of the reconstructed pore microstructures. In the second step, results from LPT were used as input to calculate the percolation threshold based on classical percolation theory. 
Table 1. Overview of Properties of Data Sets for Different Tomographic Methods

\begin{tabular}{|c|c|c|c|c|c|c|c|}
\hline Sample & $\begin{array}{l}\text { Characteristic of } \\
\text { Sample Location }\end{array}$ & Method & $\begin{array}{c}\text { Size } \\
\left(\mu \mathrm{m}^{3}\right)\end{array}$ & $\begin{array}{c}\text { Voxel } \\
\text { Size }\left(\mathrm{nm}^{3}\right)\end{array}$ & $\begin{array}{l}\text { Porosity } \\
\text { [vol. \%] }\end{array}$ & $\begin{array}{c}\text { Resolved } \\
\text { Pore Radii }(\mathrm{nm})\end{array}$ & Figures and References \\
\hline \multirow[t]{2}{*}{ BWS sandy facies } & $1=$ clay-rich zone & FIB & 278.4 & $15^{3}$ & 7.6 & $\geq 15$ & Figures $2 \mathrm{c}$ and $2 \mathrm{~d}$ \\
\hline & $2=$ carbonates & FIB & 3850.9 & $20^{3}$ & 2.5 & $\geq 20$ & Figures $2 \mathrm{a}$ and $2 \mathrm{~b}$ \\
\hline \multirow[t]{3}{*}{ BDR shaley facies } & 1a "Pressure shadow" & FIB & 331.8 & $10^{3}$ & 2.3 & $\geq 5$ & Keller et al. [2013] \\
\hline & 1b "Highly compacted" & FIB & 303.6 & $10^{3}$ & 2.8 & $\geq 5$ & Keller et al. [2013] \\
\hline & 1 & STEM & 0.1 & $2.3^{3}$ & 13.5 & $\geq 2$ & Keller et al. [2013] \\
\hline
\end{tabular}

[5] In order to account for spatial porosity fluctuations, LPT was applied to two types of reconstructed microstructures: (i) porosity in clay matrix related to samples with different clay contents and (ii) porosity in carbonate layers. LPT describes two geometric properties. The first property is the local porosity distribution, which accounts for porosity fluctuations. The second property is the local percolation probability, which gives information on connectivity fluctuations. Concerning geomaterials, this theory was used by Biswal et al. [1998] to characterize the pore space of sandstones.

[6] Results from local porosity theory can then be used to calculate the percolation threshold on the basis of classical percolation theory [Stauffer and Aharony, 1995; Hunt and Ewing, 2009; Pringle et al., 2009]. So far, the theory was not often used in studies of natural materials, but recently, the theory was successfully applied to realistic pore models of sea ice [Pringle et al., 2009] and deformed rocks [Liu and Regenauer-Lieb, 2011].

[7] Security assessments often use gas flow modeling approaches that rely on the continuum hypothesis, and thereby the existence of a representative volume element (RVE) is simply assumed. A verification of this assumption is overdue. In part, these bending questions can be addressed by a quantitative analysis of shale microstructures, which were reconstructed on the basis of tomographic methods [Keller et al., 2011; Keller et al., 2013]. If the pore structure at a specific level of microscopic magnification (i.e., resolution) possesses certain homogeneity, then it should be possible to estimate the representative volume element (RVE) for this microstructural level. In order to estimate the size of the RVE of porosity, we used the method of Kanit et al. [2003], which was developed within the geostatistical framework [Matheron, 1971]. The method provides a quantitative description of the relationships between RVE size, number of realizations, and estimation accuracy. Regarding the evaluation of tomographic methods, the statistical method used is particularly useful as it permits an error estimate and improves the sampling strategy, with respect to the chosen sample size and numbers of realizations.

[8] For this paper, we used new and previously published [Keller et al., 2013] FIB-nt data and related pore space reconstructions as a prerequisite to address the fundamental question of by what properties (e.g., porosity) gas can flow through the pore space and arrive at the other side of the system. Apart from LPT and percolation theory, we used also a two-phase flow pore-network modeling [Valvatne and Blunt, $2004]$ in combination with pore space reconstructions to discuss the microstructural control of gas flow in Opalinus clay.

\section{3-D Data}

\subsection{Analytical Methods}

[9] Sample preparation, tomographic methods (FIB, scanning transmission electron microscopy (STEM)), and image processing (segmentation and determination of morphological and topological parameters) are described in Keller et al., $[2011,2013]$. The absolute size of the analyzed volumes, voxel size, and bulk porosity related to the respective tomographic methods are documented in Table 1.

\subsection{Input Format of 3-D Data}

[10] 3-D data of pore space in Opalinus clay are available from our previous studies (Table 1) [Keller et al., 2013]. In addition, two new FIB data sets, corresponding to a sample from the sandy facies, were used in this study (Table 1). Here high-resolution tomography (with voxel sizes in the range $2-20 \mathrm{~nm}$ ) is based either on tilt series from transmission electron microscopy (STEM) or on serial sectioning experiments with focused ion beam (FIB). These techniques provide information about the pore structure, which is mainly hosted in the clay matrix and in carbonate-rich zones.

[11] The segmented images from transmission electron microscopy (TEM) and FIB tomography were transformed into binary images, in which the pores were set to white and the remaining area to black. Segmentation quality of our image processing workflow is discussed in Keller et al. [2011]. The resulting images are two-dimensional arrays of ones (i.e., white) and zeros (i.e., black). The images were then read into three-dimensional arrays of zeros and ones, in which the ones indicate the pore space. For the statistical analysis by means of local porosity theory, the voxel arrays were then regularly subdivided into cubic subarrays of $N \times N \times N$ voxels. This operation divides the analyzed volume into a regular cubic grid/lattice, in which each cell $K(x, L)$ is located at a position $x$ and has an edge length $L$ that corresponds to a certain number of voxels. Once the image stacks are subdivided into such a grid, it is possible to determine quantities (e.g., porosity and connectivity) for each cell $K(x, L)$, which are then used as input for the calculation of the local porosity distribution. Unfortunately, the data sets available from high-resolution tomography only have a limited size of the image window. Therefore, the use of nonoverlapping cells led to poor statistics, particularly for larger $L$, with strong fluctuations in the results. To overcome this problem, we used overlapping cells [see also Biswal et al., 1998]. The results below were obtained by placing a measuring cell at all $x$ corresponding to points of a cubic lattice with a lattice constant $L / 2$.

\section{Samples}

[12] The analyzed samples were taken from the Opalinus clay unit at the Mont Terri rock laboratory in northwestern Switzerland [Bossart and Thury, 2008]. This laboratory is located adjacent to the safety gallery of the Mont Terri motorway tunnel. Sedimentation of Opalinus clay occurred around 174 Myr ago in a shallow marine basin. After sedimentation, 

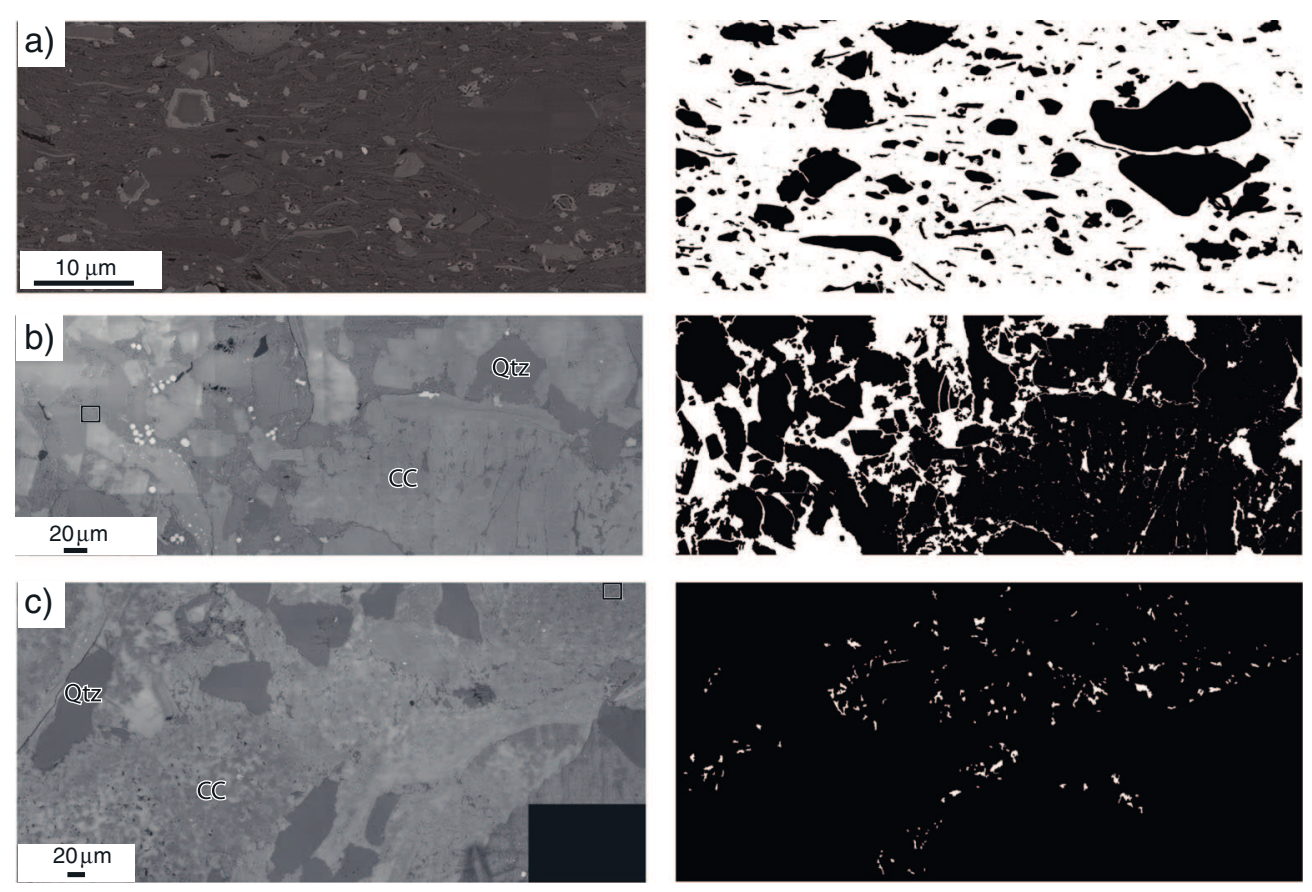

Figure 1. Large-scale SEM image of (a) shaley (sample BDR) and (b, c) sandy facies (sample BWS) of the Opalinus clay. The black rectangles in Figures $1 \mathrm{~b}$ and $1 \mathrm{c}$ mark the sites that were analyzed by FIB-nt. FIB-ntbased reconstructions of the pore spaces are depicted in Figure 2. The images on the RHS show the (white) clay content that was segmented from the images. From top to bottom, the clay contents are: 74,21 and 1 area $\%$.

the rock unit underwent two stages of burial with a maximum burial depth of about $1350 \mathrm{~m}$. Folding of the Jura mountain belt occurred between 10.5 and 3 Myr ago. The Opalinus clay can be subdivided into three main facies: shaley facies, sandy facies, and carbonate-rich sandy facies. In order to account for the microstructural variability, one of the samples (drill core BWS-H6) was taken from the sandy facies and the other (sample BDR1_oc) of the shaley facies of Opalinus clay.

\subsection{Microscale to Macroscale Structures}

[13] Panoramic scanning electron microscope (SEM) images on the scale of hundreds of microns of samples polished with broad ion beam are shown in Figure 1. At this scale, the main structural characteristics that varies between the different samples is the clay content (Figure 1).

[14] The sample from the shaley facies consists of a clayrich matrix with dispersed nonclayey grains (Figure 1a). Clay matrix content in Figure $1 \mathrm{a}$ is around 74 area $\%$. Hereafter, this microstructure is referred to as the matrixsupported microstructure. Regarding the sample of the shaley facies, the interested reader may find additional data (e.g., modal amounts of major constituents) and other microstructural analyses in Keller et al. [2013].

[15] In the case of the sample from sandy facies, the SEM images reveal a grain-supported texture where nonclayey grains are largely touching. Clay matrix content is around 21 and 1 area $\%$, respectively (Figures $1 \mathrm{~b}$ and $1 \mathrm{c}$ ).

\subsection{Nanoscale Structures}

[16] 3-D reconstructions of the pore space in the shaley facies based on FIB and STEM (i.e., voxel resolutions 10 and $2.3 \mathrm{~nm}$ ) can be found in Keller et al. [2013]. The two FIB realizations of the shaley facies correspond to particularly high and low degrees of compaction ("highly compacted zones" and "pressure shadows," respectively) (Table 1) [Keller et al., 2013]. The purpose of the two FIB realizations was to consider spatial variability in the 3 -D pore space of the clay-rich ("clay matrix") zone of the shaley facies.

[17] Regarding the new FIB data of the sample from the sandy facies, the panoramic SEM images (Figures $1 \mathrm{~b}$ and 1c) show the selected target volumes that were analyzed by FIB-nt. The corresponding 3-D reconstruction of the pore microstructure in the carbonates is displayed in Figures $2 \mathrm{a}$ and $2 \mathrm{~b}$. It must be emphasized that the $3-\mathrm{D}$ visualization may be misleading because carbonate-rich zone has porosity as low as 0.027 , and the pore space was found to be largely unconnected. The corresponding continuous pore size distribution [Münch and Holzer, 2008] is displayed in Figure 3 and shows that pore radii are in the size range of 50-300 nm.

[18] The 3-D pore structures in the clay matrix of the sandy facies are displayed in Figures $2 \mathrm{c}$ and $2 \mathrm{~d}$. The porosity in the sample of the sandy facies is around 0.076, while it is only 0.023 and 0.028 in the case of the sample of shaley facies (Figure 3 and Table 1). The PSD (pore size distribution) analyses reveal that the clay matrix of the grain-supported microstructure in the sandy facies has a much higher fraction of "larger pores" compared with the one of matrix-supported structure in the shaley facies (Figure 3). The notation "larger pores" is related to the fact that FIB-nt can resolve only a small fraction of comparatively larger pores [Keller et al., 2011]. The "smaller pores" can be captured by TEM. However, with this technique, the image window is too small for representative analysis of the clay microstructures. Finally, within the sandy facies, the pores in the clay matrix are smaller than the pores in carbonates (Figure 3). 
a)

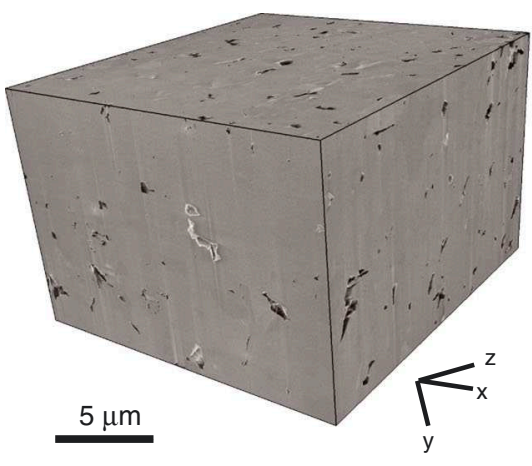

c)

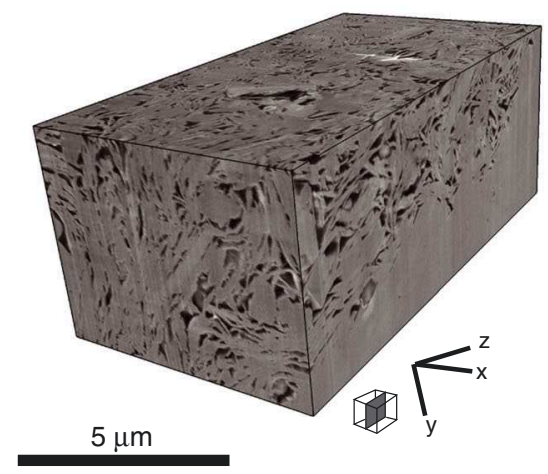

b)

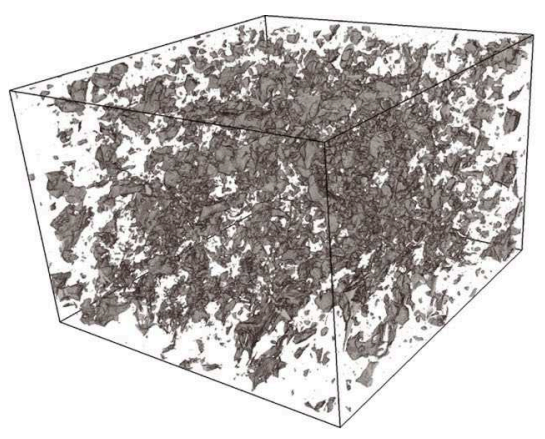

d)

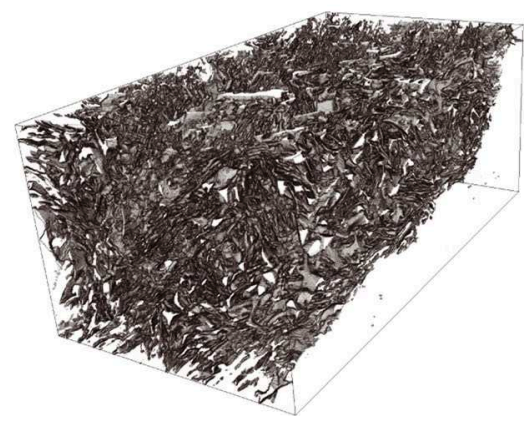

Figure 2. Reconstructed pore microstructures based on FIB-nt. See Table 1 for voxel resolution and porosity. (a) The 3-D reconstruction of the analyzed volume of carbonates of sandy facies based on SE (secondary electron) images. (b) Pores in carbonates of sandy facies. (c) The 3-D reconstruction of the analyzed volume of clay-rich zone of sandy facies based on SE images. (d) Pores in clay matrix of sandy facies. The orientation of the bedding plane is indicated by the small cube with a gray plane.

\section{Local Porosity Analyses}

\subsection{Local Porosity Theory}

[19] To characterize the heterogeneity of the material and to evaluate the representativeness of 3-D data from tomographic methods, which are used to study the pore structure, we followed the approach outlined in Hilfer [1991], Biswal et al. [1998], Hu and Stroeven [2005], and Hilfer and Helmig [2004]. Based on the gridded 3-D array (see above), the local porosity $\phi(x, L)$ can then be measured in each cubic cell $K(x, L)$ by counting the numbers of pore voxels. The local porosity distribution is then defined as

$$
\mu(\phi, L)=\frac{1}{m} \sum_{i=1}^{m} \delta\left(\phi-\phi\left(x_{i}, L\right)\right),
$$

where $m$ is the number of placements of measurement cells and $\delta(t)$ denotes the delta function (sometimes called the Dirac delta function). The function $\mu(\phi, L)$ is a measure for the probability to find the local porosity $\phi$ in a cell with side length $L$. Here the local porosity distribution is evaluated for five to seven different cell sizes, which represent subsamplings from the same set of tomographic data.

[20] Regarding permeability and material transfer, the question whether or not a pore space percolates is fundamental. A cell $K(x, L)$ is considered as percolating in $x$ direction if there exists a continuous path along the pore space which connects the two faces perpendicular to the $x$ direction.
This definition is also applied to the other directions. The following formulation is used as an indicator for percolation.

$$
\Lambda_{\alpha}(x, L)=\left\{\begin{array}{l}
1 \text { if } K(x, L) \text { percolate in } \alpha \text { direction } \\
0 \text { otherwise }
\end{array}\right.
$$

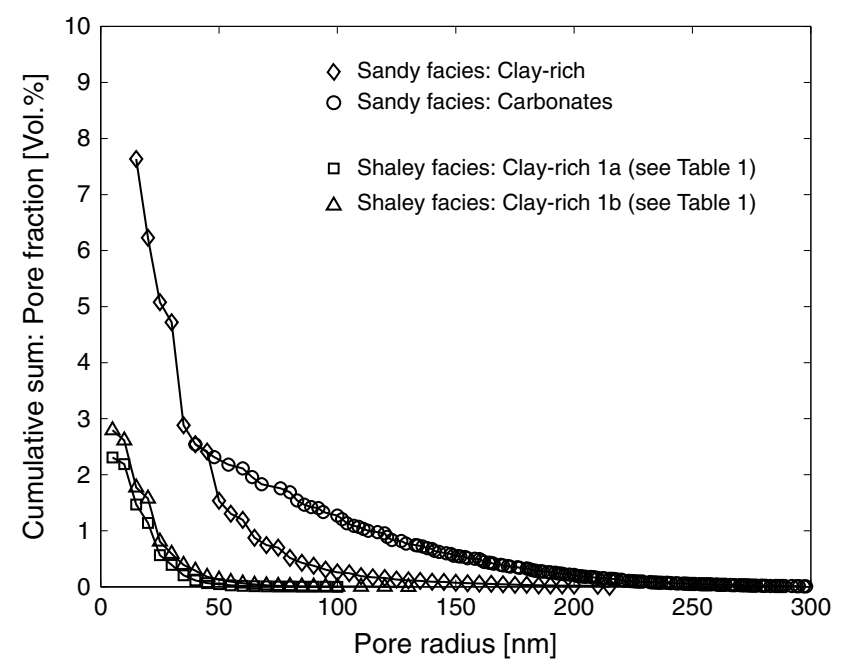

Figure 3. Compilation of continuous pore size distributions for different materials in Opalinus clay which are based on the 3-D data from FIB tomography. See text for further discussion. 
Table 2. Legend of Index $\alpha$ of Local Probabilities $\lambda \alpha(\phi, L)$

\begin{tabular}{lc}
\hline Index $\alpha$ & Meaning \\
\hline$x$ & $x$ direction \\
$y$ & $y$ direction \\
$z$ & $z$ direction \\
3 & $(x \wedge y \wedge z)$ direction \\
1 & $(x \vee y \vee z)$ direction \\
0 & $\neg(x \vee y \vee z)$ direction \\
\hline
\end{tabular}

[21] The meaning of $\alpha$ direction is defined in Table 2. As an example, $\Lambda_{3}$ indicates that a cell can be traversed in all three directions, while $\Lambda_{1}$ indicates that there exists at least one direction along which the cell is percolating. $\Lambda_{0}$ indicates a blocking cell.

[22] The local percolation probability is then defined by the following:

$$
\lambda_{\alpha}(\phi, L)=\frac{\sum_{i-1}^{m} \Lambda_{\alpha}\left(x_{i}, L\right) \cdot \delta\left(\phi-\phi\left(x_{i}, L\right)\right)}{\sum_{i=1}^{m} \delta\left(\phi-\phi\left(x_{i}, L\right)\right)},
$$

where $\lambda_{\alpha}(\phi, L)$ is the fraction of cells with local porosity $\phi$ and side length $L$ that allow percolation in $\alpha$ direction. A pore space is fully connected if $\lambda_{3}=1$. In practice, the Dirac delta function $\delta(t)$ is seen as a kind of limit of a sequence of functions having a tall spike at the origin. Different approximating functions can be defined. Here we used the following function:

$$
\delta(t)=\frac{1}{2 \sqrt{\pi \zeta}} e^{-\frac{t^{2}}{4 \zeta}} \quad \text { with } \zeta=0.00001
$$

[23] When plotted against $t$, the above function appears as a narrow spike at $t=(\phi-\phi(x, L))=0$ and vanishes outside a very narrow range symmetric around $t=0$. Thus, if a certain local porosity is found within a cell, the function acts as a kind of an "impulse response function" and, in combination with equation (1), collects the response of local porosities and yields a continuous probability function with varying local porosity. Setting the $\zeta$ parameter to the above value ensures that the continuous function still reflects small porosity fluctuations. The determination of $\Lambda_{\alpha}(\phi, L)$ requires the determination of the connected pore regions in the measuring cell. The Matlab $^{\circledR}$ function "bwlabeln" labels the connected regions and thus allows determining whether these regions connect the faces of the measuring cell or not. "bwlabeln" basically scans the image columnwise and makes each connected pore voxel a node in a graph and then combines the graphs, which are connected (see http://blogs.mathworks.com/steve/2007/ 05/25/connected-component-labeling-part-6/).

[24] The local porosity distributions displayed in Figure 4 correspond to the porosity in the carbonates and clay matrix, which are evaluated based on the FIB data (Table 1). With increasing $L$, the $\mu$ curves change from a wide distribution to
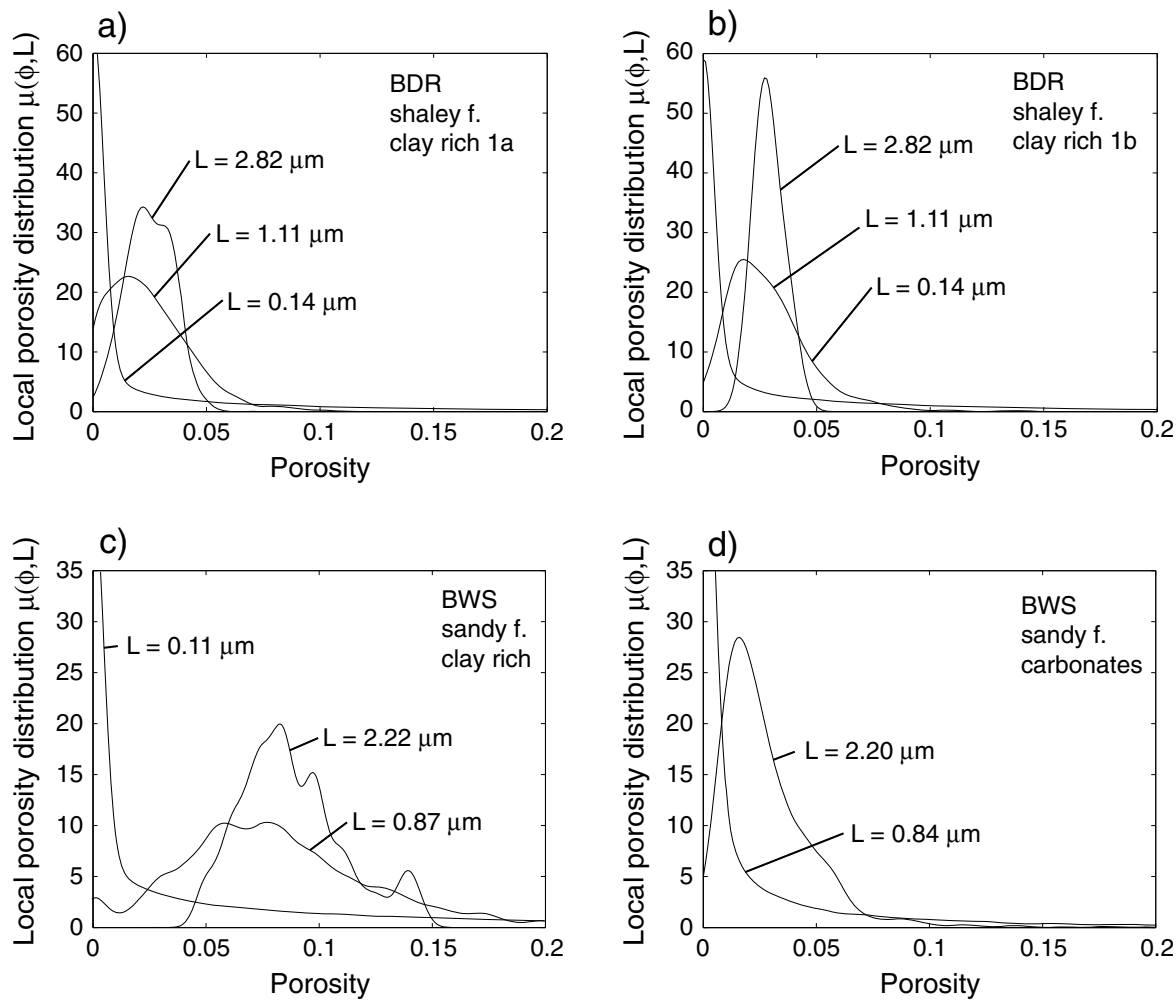

Figure 4. Local porosity distributions calculated for different length $L$ of measuring cell as obtained by analyzing images from FIB tomography. ( $a, b)$ Shaley facies of Opalinus clay (see Table 1 for source of the used data). (c) Sandy facies of Opalinus clay (corresponding to microstructure Figure 2b). (d) Carbonates of sandy facies of Opalinus clay (corresponding to microstructure Figure 2a). 
a)

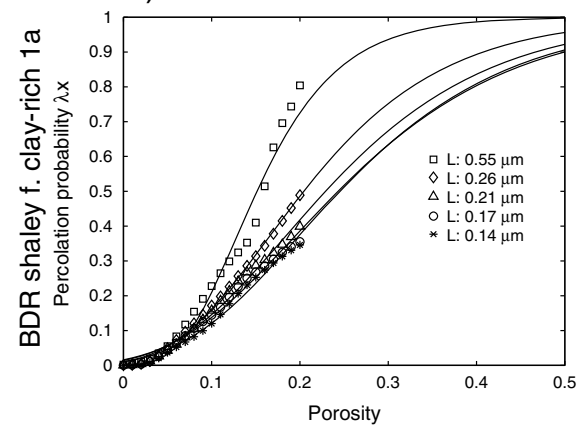

d)

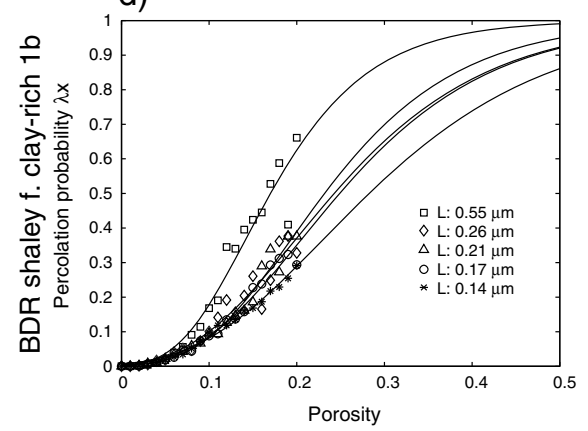

g)

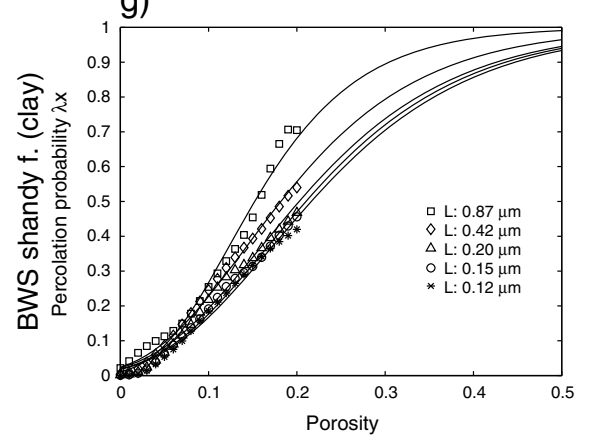

b)

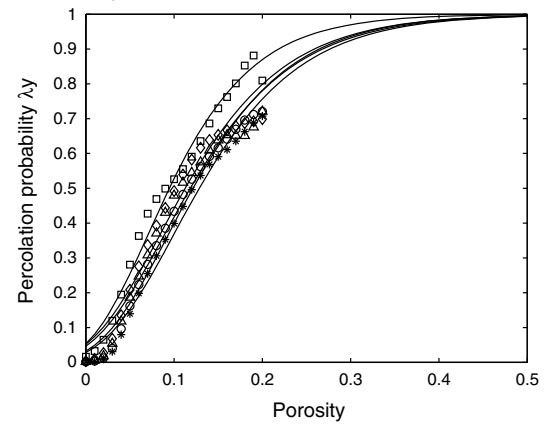

e)

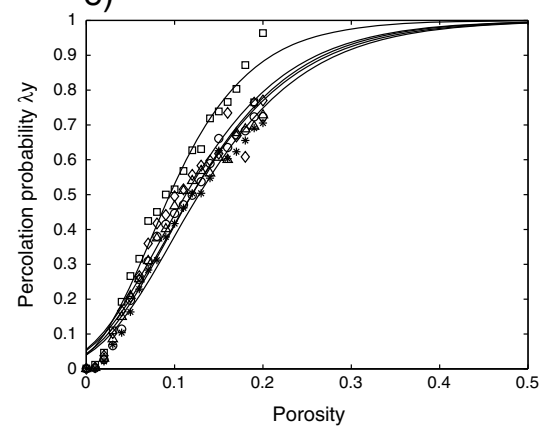

h)

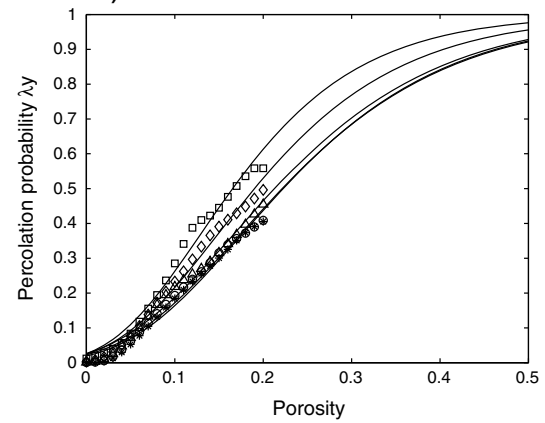

c)

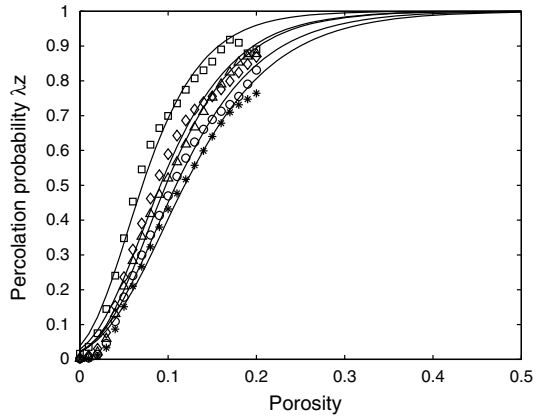

f)

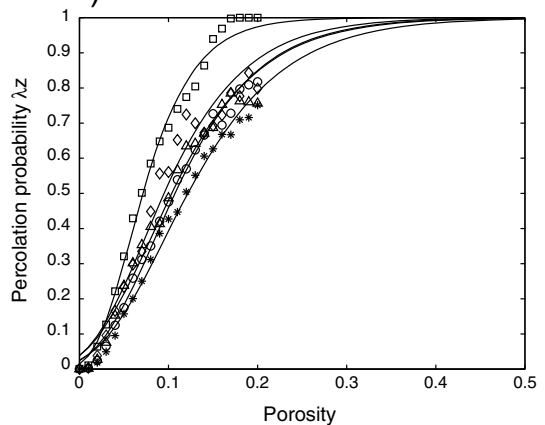

i)

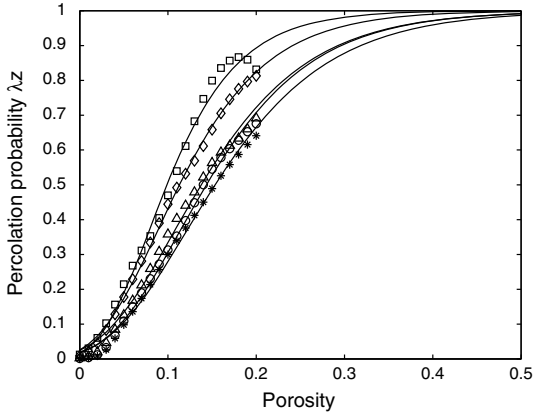

Figure 5. Percolation probabilities $\lambda_{\alpha}(\phi, \Lambda)$ along different directions $\left(\lambda_{x}, \lambda_{y}\right.$, and $\left.\lambda_{z}\right)$. Symbols are computed values and lines represent smooth interpolations. (a-f) Shaley facies of Opalinus clay (see Table 1 for source of the used data). (g-i) Sandy facies of Opalinus clay (corresponding to microstructure Figure 2b).

a distribution with a single peak at the position of the bulk porosity. Development of a single peak occurs for cells with edge lengths $L>\sim 2 \mu \mathrm{m}$. This means that at this length scale, there is a high probability to find a local porosity that equals the bulk porosity, which in turn can only be true if the pore space, at this length scale, possesses a certain degree of homogeneity. In addition, the function component at the origin decreases with increasing $L$ (Figure 4). All these results indicate that similarities between local geometries increase with increasing $L$. It also indicates that the porosity in the clay matrix and carbonates possesses a certain degree of homogeneity on the few micron scale.

\subsection{Local Percolation Analyses}

[25] Calculated $\lambda_{\alpha}(\phi, L)$ related to porosity in the clay matrix in both shaley and sandy facies are displayed in Figure 5. The smooth curves in Figure 5 are fitted curves, which were used as input for the calculation of the percolation threshold (see below). The pores in the carbonate-rich zones are very poorly connected, and hence no percolation analysis is shown in Figure 5 for the carbonates.
[26] One notices that the clay matrix is anisotropic with respect to the connectivity. Sample BDR (shaley facies) has a higher connectivity in $y$ and $z$ directions compared to the $x$ direction (i.e., higher percolation probabilities in $y$ and $z$ directions). Note that both pore structures of sample BDR show similar percolation probabilities (Figure 5). Sample BWS (sandy facies, clay-rich zone) has a higher connectivity in $z$ direction. These results reflect the anisotropy of the clay samples, which both have a bedding parallel to the $y z$ plane. All this indicates a higher connectivity in the bedding plane when compared to the direction perpendicular to the bedding.

[27] The difference in connectivity behavior is also documented by the function [e.g., Biswal et al., 1998]

$$
P_{\alpha}(L)=\int_{0}^{1} \mu(\phi, L) \lambda_{\alpha}(\phi, L) \mathrm{d} \phi,
$$

which gives the total fraction of the cells percolating in $\alpha$ direction (Figure 6). The cell sizes are indicated by the 
a)

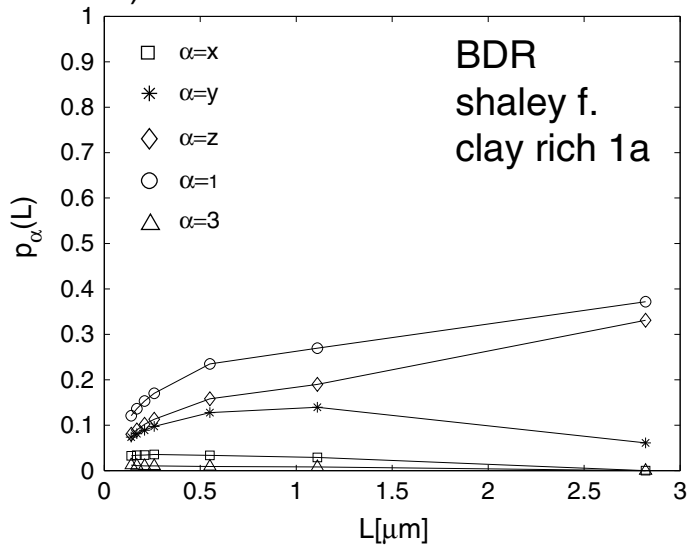

c)

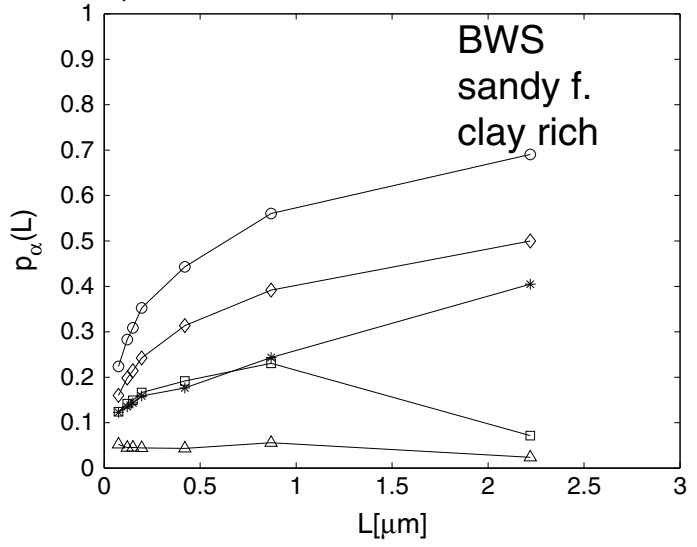

b)

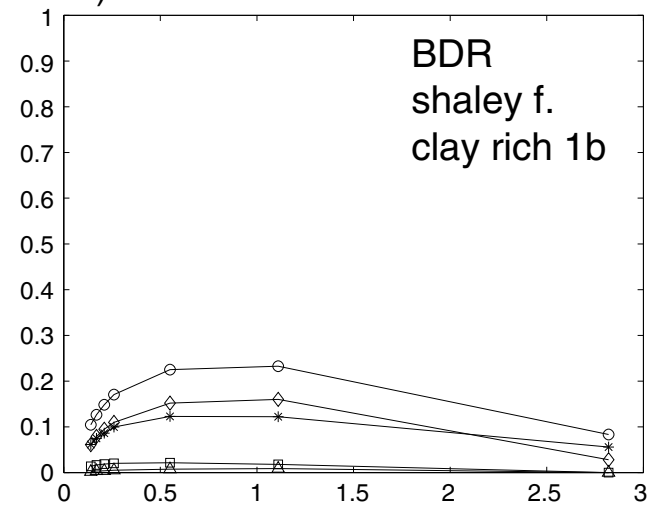

d)

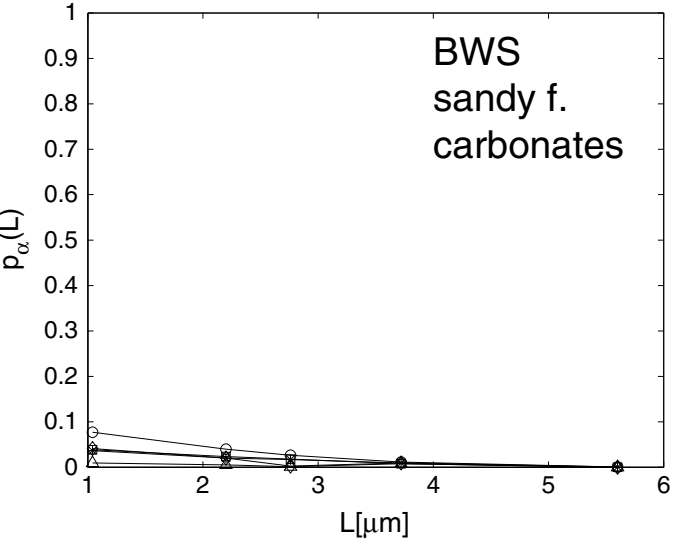

Figure 6. The total fraction of percolation cells $p_{\alpha}(L)$ with $\alpha=1,3, x, y, z$ for the pores in (a, b) clay matrix of shaley facies, in (c) clay matrix of sandy facies, and (d) in carbonates of sandy facies.

symbols in Figure 6. The calculations show that pores in the carbonates are very poorly connected, and indications for connectivity anisotropies are absent. Thus, long-range percolation along the pore space of the carbonates is rather unlikely.

[28] On the contrary, the pore space in the clay matrix is better connected, and the corresponding percolation is anisotropic with $p_{z} \vee p_{y}>p_{x}$, whereby the $y z$ plane is parallel to the bedding plane. Regarding the sample from the shaley facies, connectivity decreases with increasing $L$ (Figures $6 \mathrm{a}$ and $6 \mathrm{~b}$ ), which reflects the fact that the pore structure is poorly connected for larger $L$ (see below). The pore space of the sample from the sandy facies is better connected when compared to the one of the shaley facies as it might be expected based on the higher porosity of this sample. The plots give also an indication on the fractions (i.e., probabilities) of percolating sites in a critical site percolation network model (see below). Because $p_{\alpha}$ depends on lattice constant $L$, the plot also provides information on how to relate the lattice constant of such a model to the length scale of the real rock.

\section{Percolation Threshold}

[29] The critical porosity at which one can expect a percolating pore network is a crucial parameter. To determine the percolation threshold on the basis of tomographic data, several approaches are used [Pringle et al., 2009; Liu and RegenauerLieb, 2011]. Here we used finite-size scaling in combination with the results obtained from local percolation analysis
[Pringle et al., 2009]. In addition, we applied basic morphological image processing techniques directly to the voxel data in order to determine the percolation threshold of the nanoporosity in the sample [see Liu and Regenauer-Lieb, 2011]. Both methods are described in the two following sections.

\subsection{Percolation Threshold Based on Finite-Size Scaling}

[30] The following procedure is based on classical percolation theory. This theory describes the connectivity between a large numbers of objects (e.g., pore objects). In numerical lattice simulations, the most fundamental variable is $p$, which for site percolation problems is the fraction of lattice sites that are occupied and allow flow trough. In continuum percolation, $p$ may be a fractional volume, for example, the porosity $\phi$. The most important value of $\phi$ is $\phi_{c}$, which represents the critical porosity at which percolation occurs. It is important to note that the critical volume fraction $\phi_{c}$ may be different from the critical site fraction $p_{c}$, because the percolation threshold of a real pore structure is essentially a function of the space geometry [e.g., Garboczi et al., 1995; Hunt and Ewing, 2009]. Therefore, in the present study, $\phi$ is preferred, and $p$ is only used in the context of lattice percolation problems.

[31] The calculated percolation probability $\lambda_{\alpha}(\phi, L)$ can be regarded as the probability that a sample with a given porosity $\phi$ and size $L$ percolates along a direction $\alpha$ [Stauffer and Aharony, 1995]. For infinite (i.e., large $L) L$ and if $\phi$ is below a critical value $\phi_{c}$, there is no percolating path across the 
a)

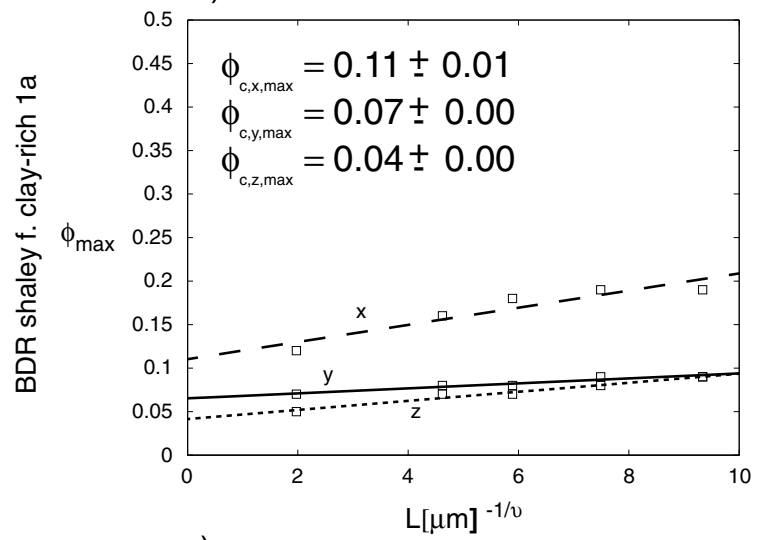

c)

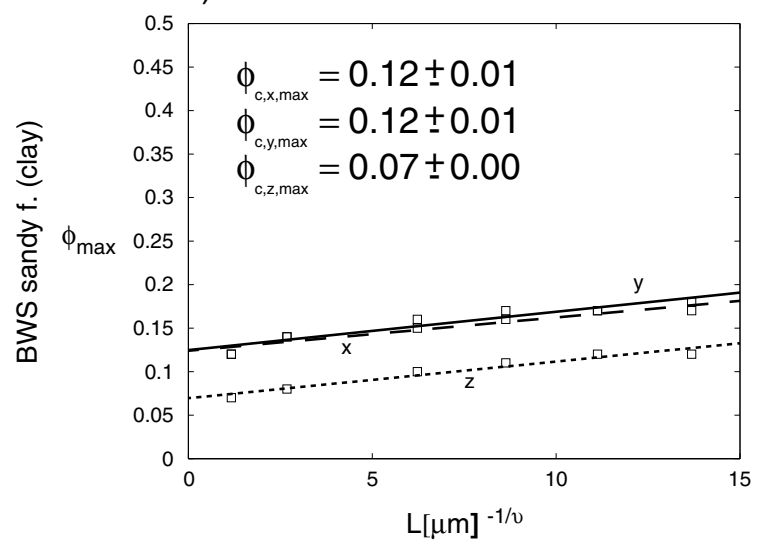

b)

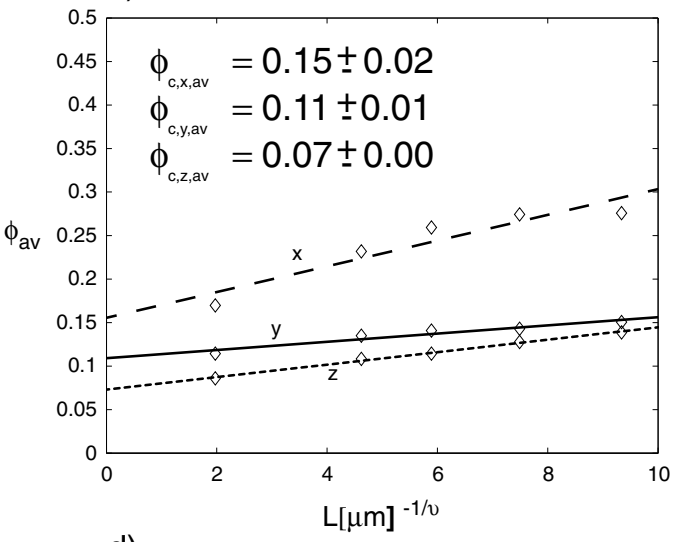

d)

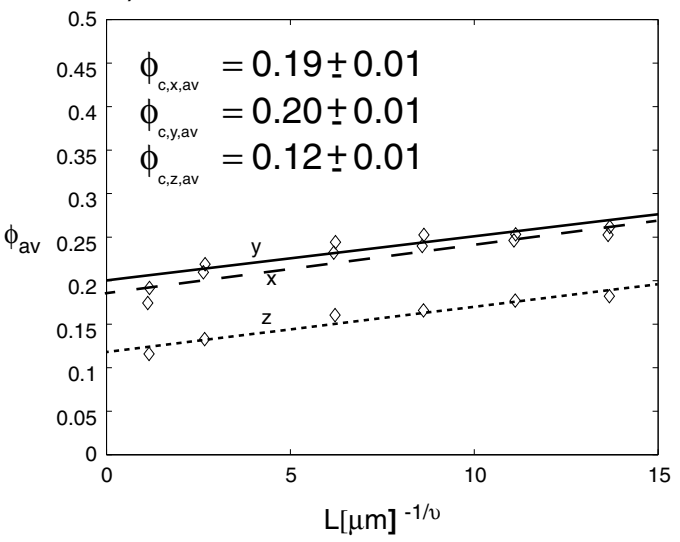

Figure 7. $\phi_{\max }$ and $\phi_{\mathrm{av}}$ versus $\mathrm{L}^{-1 / v}$. (a and b) Pores in clay matrix of shaley facies. The plot corresponding to the second FIB-nt data set of the shaley facies (Table 1) is similar to Figures $7 \mathrm{a}$ and $7 \mathrm{~b}$ and is thus not shown. (c and d) Pores in clay matrix of sandy facies. Lines are least squares fits of which intercept values are given in the plots and are considered as percolation thresholds.

sample, whereas for small $L$, it is possible to find a percolating path even if $\phi<\phi_{c}$. This is the typical situation for the porosity in the clay matrix where we have $\lambda_{\alpha}\left(\phi<\phi_{c}\right)=0$ for the sample size that can be analyzed by FIB-nt. For subvolumes, however, we found $\lambda_{\alpha}(\phi, L)>0$. The question now is how can we determine the critical value $\phi_{c}$ (i.e., percolation threshold) for a sample of infinite size from the calculated $\lambda_{\alpha}(\phi, L)$ curves related to small values of $L$. Regarding percolation theory, this can be done by a technique known as finite scaling [Stauffer and Aharony, 1995]. For an infinite sample, $\lambda_{\alpha}(\phi, \infty)$ is a step function with $\lambda_{\alpha}\left(\phi<\phi_{c}\right)=0$ and $\lambda_{\alpha}\left(\phi>\phi_{c}\right)=1$. When considering smaller samples with statistical methods, one can observe a range of porosities which represent the percolation threshold. This transition range widens as sample size decreases (Figure 5). Figure 5 also shows that the porosity range of our samples does not cover full transition from $\lambda_{\alpha}(\phi, L)=0$ to 1 for all $L$. To improve the covered porosity range and to avoid influence of local percolation fluctuations, we analyzed smooth curves fit to our data [see also Pringle et al., 2009]. The derivation $\mathrm{d} \lambda / \mathrm{d} \phi$ is essentially the probability of the first occurrence of a percolating sample with porosity $\phi$ [Stauffer and Aharony, 1995]. The effective percolation threshold can be defined as the porosity with maximum probability $\phi_{\max }$ for the first occurrence. For this purpose, $\phi_{\max }$ can be found by calculating the point of

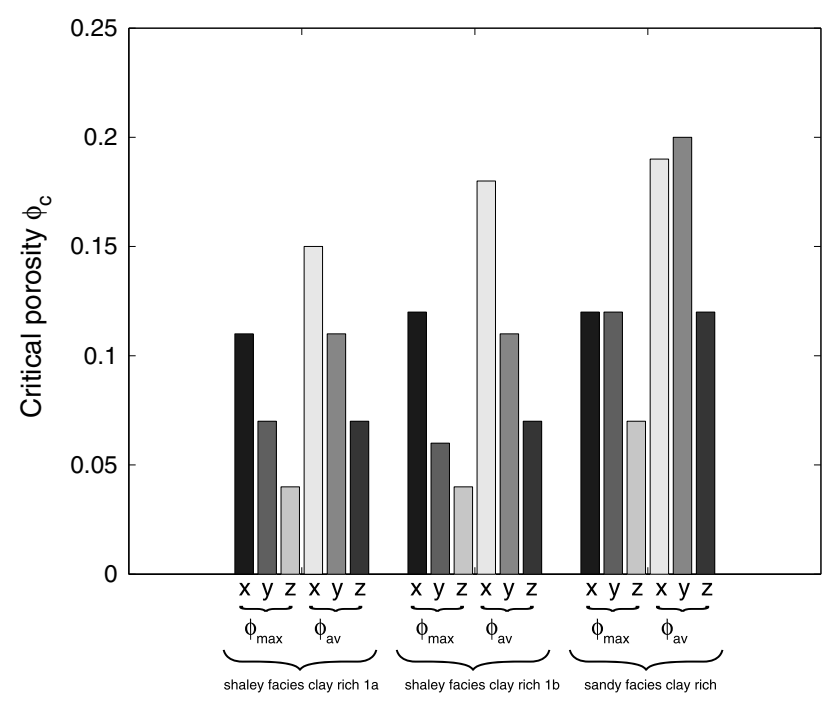

Figure 8. Bar plot shows a compilation of the calculated percolation thresholds for all samples. The values related to different percolation directions $(x, y, z)$ are grouped according to the applied scaling scheme (i.e., $\phi_{\max }$ and $\left.\phi_{\mathrm{av}}\right)$. 
Table 3. Porosity and Percolation of Inflated Pore Structure Models ${ }^{\mathrm{a}}$

\begin{tabular}{lccccc}
\hline Operation & Porosity (vol. \%) & Percolation & Absolute Permeability $\|$ to Bedding $\left(\mathrm{m}^{2}\right)$ & Gas Entry Pressure (MPa) & Gas Emergence Pressure (MPa) \\
\hline 0 & 2.3 & 0 & - & - & - \\
+1 & 4.38 & $y$ & $4.2 \mathrm{E}-21$ & 2.2 & - \\
+2 & 6.71 & $y$ & $3.2 \mathrm{E}-19$ & 1.5 & 4.5 \\
+3 & 9.23 & $y, z$ & $6.2 \mathrm{E}-18$ & 1.3 & 7 \\
+4 & 11.85 & $x, y, z$ & - & - & - \\
+5 & 14.57 & $x, y, z$ & - & - & - \\
+6 & 17.35 & $x, y, z$ & - & - & - \\
+7 & 20.19 & $x, y, z$ & & & - \\
\hline
\end{tabular}

\footnotetext{
${ }^{\mathrm{a}}$ In the operation column, 0 denotes the original model, and positive numbers indicate the number of inflation steps. Columns 3-6 are results related to gas
} transport that were obtained by pore network modeling (see discussion).

inflection of $\lambda(\phi)$ (i.e., porosity with maximum of $\mathrm{d} \lambda / \mathrm{d} \phi$ ) [Stauffer and Aharony, 1995; Pringle et al., 2009]. The point of inflection converges to $\phi_{c}$ as [Stauffer and Aharony, 1995]:

$$
\phi_{\max }-\phi_{c} \propto L^{-1 / v},
$$

where $v$ is the critical exponent of the correlation length, which has a universal value depending only on the system dimension, and in 3-D, we have $v=0.88$ [Bunde and Havlin, 1995; Pringle et al., 2009]. Figure 7 shows a plot of numerically calculated $\phi_{\max }$ versus $L^{-1 / v}$.

[32] $L^{-1 / v}$ decreases with increasing $L$, which implies that for $L \rightarrow \infty$, the value for the infinite size limit is given by the vertical axis intercept [Pringle et al., 2009]. A linear least squares fit yielded intercept values for percolation in different transport directions (Figures 7 and 8), and all calculated percolation thresholds are compiled in Figure 8.

[33] A similar behavior is shown by $\phi_{\mathrm{av}}$, the average porosity at which a percolating cluster appears for the first time. This parameter is defined by Stauffer and Aharony [1995]

$$
\phi_{\mathrm{av}}=\int_{0}^{1} \phi\left(\frac{\mathrm{d} \lambda}{\mathrm{d} \phi}\right) \mathrm{d} \phi .
$$

[34] Then, a similar scaling scheme (i.e., $\phi_{\mathrm{av}}-\phi_{\mathrm{c}}=L^{-1 / v}$ ) as for $\phi_{\max }$ is applied [Pringle et al., 2009]. Numerically calculated values of $\phi_{\mathrm{av}}$ are plotted versus $L^{-1 / v}$, and a linear least squares fit yielded intercept values for the different percolation directions (Figures 7 and 8).

[35] Concerning the sample from the shaley facies and depending on the applied scaling scheme, the calculations yielded values for the percolation threshold in $z$ direction $\phi_{c, z}$ and $y$ direction $\phi_{c, y}$ that range between $0.04-0.07$ and $0.07-0.11$, respectively (Figure 8 ). Percolation threshold in $x$ direction is higher and ranges between 0.11 and 0.18 . Almost similar values were obtained for the two pore microstructures corresponding to the sample of the shaley facies (Figure 8). Since the $y z$ plane of both samples is parallel to the bedding plane, the results indicate an anisotropic percolation threshold with a lower percolation threshold within the bedding plane. The calculated $\phi_{c}$ values are higher than the porosity of the samples from the shaley facies (Table 1). This likely indicates that the resolved pore space with pore radii exceeding $5 \mathrm{~nm}$ is not connected in the shaley facies of Opalinus clay.

[36] The sample from the sandy facies shows a similar behavior but with a lesser anisotropy in percolation threshold, which might be related to a reduced compaction of clay-rich zones in grain-supported microstructures. For this sample, a lower percolation threshold is only found in $z$ direction, whereas $y$ and $x$ directions have similar thresholds. The calculated $\phi_{c}$ values (i.e., $\sim 0.07$ ) in $z$ direction are close to the resolved porosity (Table 1), which indicates long-range connectivity of pores with radii $>15 \mathrm{~nm}$ within the clay matrix of the sandy facies. Note that the found thresholds are restricted to the pore spaces that can be resolved by FIB-nt (i.e., with a resolution of 10-20 nm; see Table 1).

\subsection{Percolation Threshold Based on Morphological Techniques}

[37] The main purpose for using this approach is to test if the results agree with the ones obtained on the basis of finite scaling. The approach was recently proposed by Liu and Regenauer-Lieb [2011]. The authors applied basic

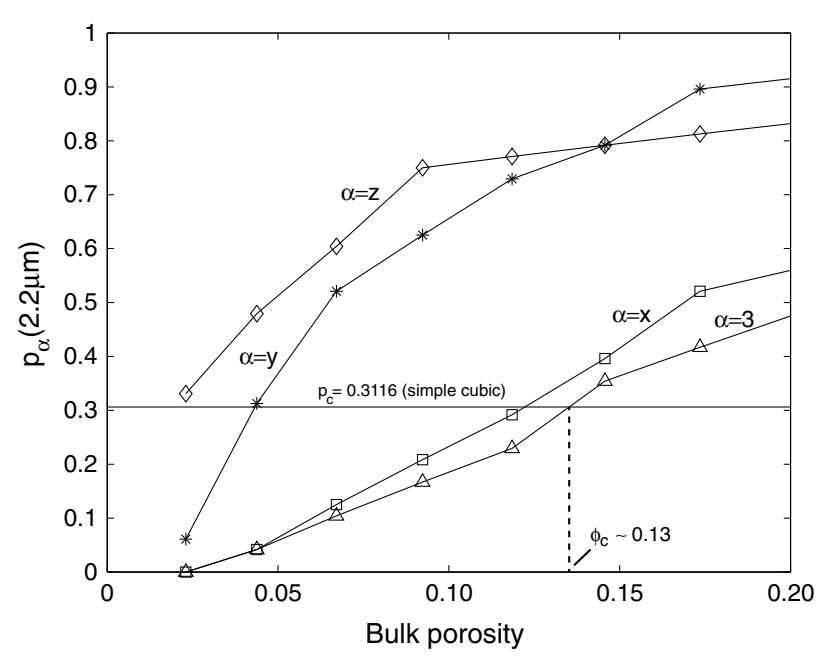

Figure 9. $p_{\alpha}(L)$ versus bulk porosity. The plot was constructed by morphologically inflating the complete FIBbased pore structure of the sample from the shaley facies. The bulk percolation threshold of the inflated models is in the $0.09-0.12$ porosity range (Table 3 ). After each inflating step, the volume was subdivided into regular grid and the fraction of percolating cells $(L=2.2 \mu \mathrm{m})$ was calculated and plotted versus porosity of the model. The theoretical percolation threshold $p_{c}$ for numerical site percolation in a simple cubic lattice is 0.3116 . The intercept between $p_{c}=0.3116$ and $p_{3}(L)$ yields a porosity of about 0.13 and may be regarded as the critical porosity for bulk percolation. This is in good agreement with the percolation threshold determined by successively inflating the pore structure. 
(a)

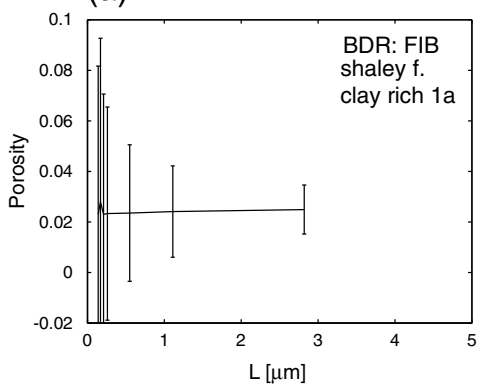

d)

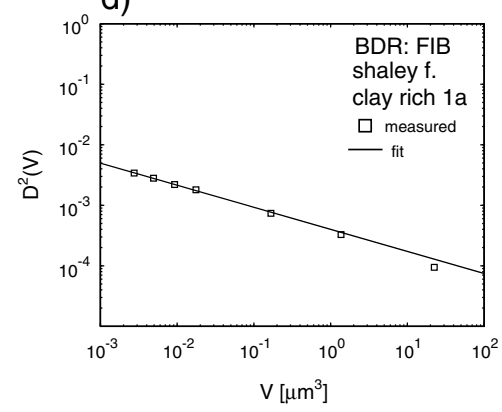

(b)

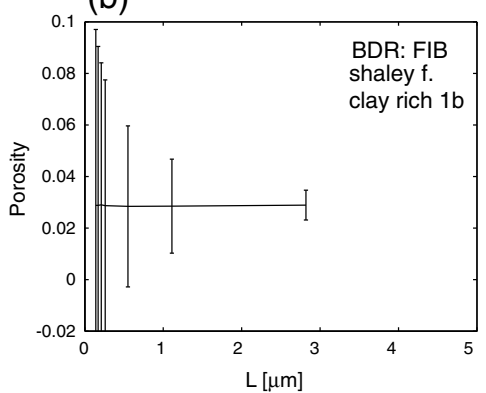

(e)

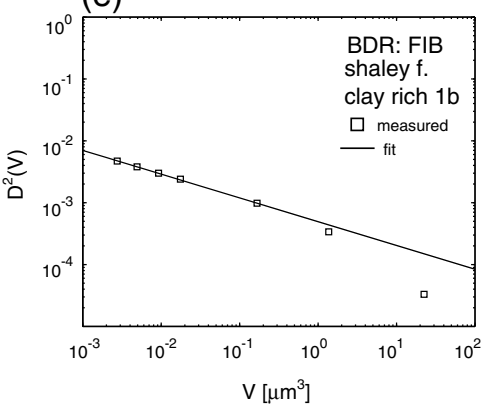

c)
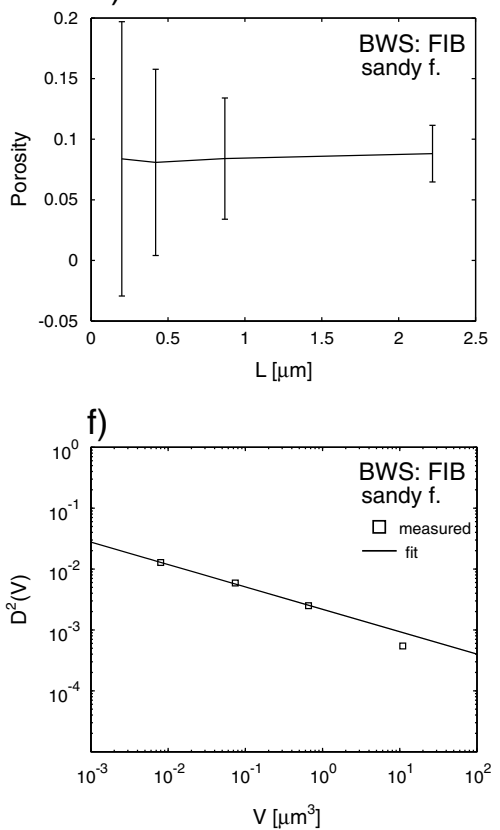

Figure 10. (a, b, c) Mean value of porosity and intervals of confidence versus size of measuring cells $L$ and $(d, e, f)$ variances of porosities versus volume of measuring cells. Shaley facies of Opalinus clay (in Figures 10a and 10b) (see Table 1 for source of the used data). Sandy facies of Opalinus clay (in Figure 10c) (corresponding to microstructure Figure 2b).

morphological image processing techniques to the voxel data. The pore voxel clusters were either deflated or inflated, which leads to a shrinkage or an expansion of the segmented pore space. Concerning the nanoporosity in the clay matrix, we inflated (i.e., expanded) the pore space step by step so that previously isolated clusters start to overlap. The expansion is performed until a pore network is formed that connects opposite sides of the analyzed volume. During one step, the boundary of the pore space moves one voxel outwards, which increases the porosity and eventually connects the nearest clusters. In this way, we obtain a sequence of pore space models, which are derived from the original model. The models have increasing porosity but preserve a structure similar to the original model (see Liu and Regenauer-Lieb [2011] for more information). For the sequence of models with different porosities, the percolation is then analyzed by applying equation (2) in order to test if the bulk model percolates or not. In the case of the BRD sample (shaley facies), porosities and percolation results are listed in Table 3. Regarding the whole volume that was analyzed by FIB-nt, continuous paths which connect all three pairs of opposite sides are created (i.e., percolation along all three directions (i.e., $x, y, z)$ ) for porosity $\phi_{c}$ in the $0.12-0.15$ range (Table 3 ). Onset of percolation in the bedding plane (i.e., $y z$ plane) occurs in $0.06-0.10$ porosity range. Note that this method yields a range of values because it cannot create models with continuous values of porosity. The obtained values agree well with the ones obtained on the basis of finite scaling. Interestingly, the determined bulk percolation threshold of porosity (i.e., $\phi_{c} \sim$ $0.12-0.15$ ) is similar to the one obtained for the clay matrix [Robinet et al., 2012], which suggests similar space geometries in shales on different length scales.

[38] In addition, we calculated the fraction of percolating cells $p_{\alpha}(L)$ for each model. Figure 9 shows a plot $p_{\alpha}(L)$ versus bulk porosity of the models. $L$ was set to $2.2 \mu \mathrm{m}$ at which value the pore space of the original model possesses a certain degree of homogeneity. Here we have used a simple cubic lattice and in such case $p_{c}$ approaches a theoretical value of 0.3116 if the system size goes to infinity [e.g., Stauffer and Aharony, 1995]. The theoretical value $p_{c}$ is plotted in Figure 9 together with the measured $p_{\alpha}$ versus the corresponding porosities. $p$ should not be confused with the porosity since it is by definition the number of occupied sites, or in the context of local porosity theory, it is the fraction of cells

Table 4. Values of Volume Fractions of Pores and Clay Content, Integral Range A3, and Coefficient $\alpha$ for Different Data Sets Obtained From Different Tomographic Methods

\begin{tabular}{|c|c|c|c|c|c|c|}
\hline Sample & Characteristic of Sample Location & Method & Porosity & Integral Range $A 3\left(\mu \mathrm{m}^{3}\right)$ & Coefficient $\alpha$ & Figures and References \\
\hline \multirow[t]{2}{*}{ BWS } & 1 clay-rich zone & FIB & 0.076 & 0.031 & 0.37 & Figures $2 \mathrm{c}$ and $2 \mathrm{~d}$ \\
\hline & 2 carbonates & FIB & 0.027 & 0.087 & 1.45 & Figures $2 \mathrm{a}$ and $2 \mathrm{~b}$ \\
\hline \multirow[t]{3}{*}{ BDR } & 1a "Pressure shadow" & FIB & 0.023 & 0.018 & 0.36 & Keller et al. [2013] \\
\hline & 1b "Highly compacted" & FIB & 0.028 & 0.018 & 0.38 & Keller et al. [2013] \\
\hline & 1 & STEM & 0.13 & 0.014 & 0.30 & Keller et al. [2013] \\
\hline
\end{tabular}




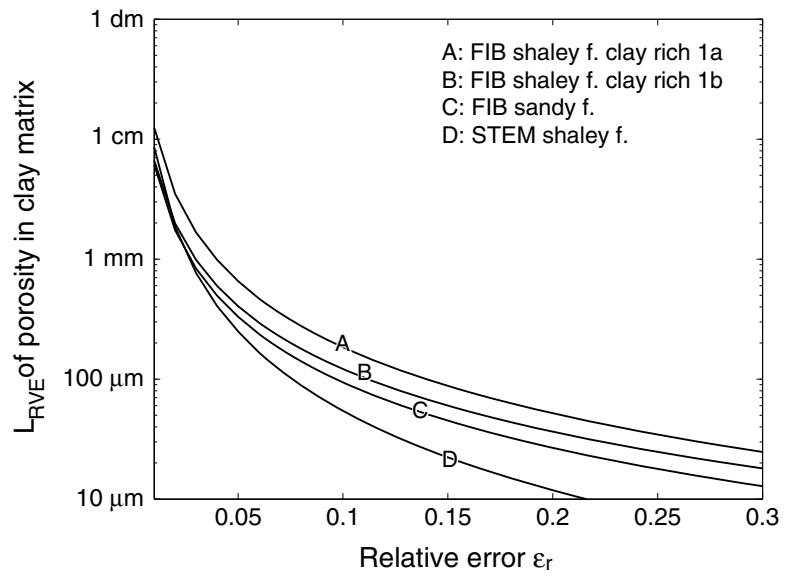

Figure 11. Size of RVE versus relative error $\varepsilon_{r}$ (calculated with $N=1$ ). RVE of porosity calculated for different microstructures and methods.

$p_{3}$ that allow percolation in all three directions. In Figure 9, the intercept of the theoretical value $p_{c}=0.3116$ with the measured function of $p_{3}$ provides the critical percolation threshold in terms of porosity, where $\phi_{c} \sim 0.13$.

\section{RVE of Porosity}

[39] Regarding the modeling of transport through rock units on a large scale, an equivalent continuum approach is commonly used [e.g., Bear, 1993]. Thereby, the porous medium is characterized by a representative volume element RVE. The RVE can be imagined as a cube in 3-D space whose properties correspond to the ones of the bulk rock. Therefore, the RVE represents the bulk rock mass in transport models on a large scale. Note that the RVE must not necessarily be the same for all parameters. Local porosity analysis shows that porosity possesses a certain spatial homogeneity on the scale of a few micrometers. However, at this length scale, the mean porosity is still related to an error, which decreases asymptotically with increasing $L$ (Figures 10a, 10b, and 10c). In addition, the mean porosity does not vary much in dependence of $L$ (Figures 10a, 10b, and $10 \mathrm{c})$. These results indicate that the true mean value of porosity can be predicted from a sample size that is smaller than the RVE and that only a sample of infinite size will produce an error-free measurement. In this way of thinking, a realistic size of RVE (i.e., not too large), which can also be used for macroscopic modeling, should be calculated for an acceptable error. In what follows and in order to calculate the size of the RVE for a given error, we used the method which is outlined by Kanit et al. [2003, and references therein]. Based on classical sample theory, the relative error on the exact mean value of porosity $M$ (or volume fraction in general), obtained from $N$ independent realizations of volume $V$, is given by the following:

$$
\varepsilon_{r}=\frac{2 D_{\phi}(V)}{M_{\phi} \sqrt{N}},
$$

where $D(V)$ is the standard deviation (i.e., the square root of variance). Following Kanit et al. [2003], the variance of porosity is given by the following:

$$
D_{\phi}^{2}(V)=\frac{M_{\phi}\left(1-M_{\phi}\right) A_{3}}{V^{\alpha}},
$$

where $A_{3}$ is referred as to the integral range which gives the information of the domain size of the pore structure for which the porosity in the measured volume $V$ has a good statistical representativity. If we plot the variance of porosity versus $L$ in a log-log plot, the points are arranged on a straight line with a slope $\neq-1$ (Figure 10). According to Lantuejoul [1991], this points to an infinite integral range, and because coefficient $\alpha$ controls the slope of the line, we have $\alpha \neq 1$. In such a case, a power law in the form of equation (9) with $\alpha \neq 1$ is proposed [Lantuejoul, 1991; Kanit et al., 2003]. In the case of a finite integral range, the slope is -1 with $\alpha=1$. Using equation (8) in equation (9) gives an expression for the smallest volume with a given relative error $\varepsilon_{r}, N$ realization and the true mean value $M$ :

$$
V\left(N, \varepsilon_{r}\right)=\left(4 \frac{\left(1-M_{\phi}\right)}{M_{\phi}} \frac{A_{3}}{N \varepsilon_{r}^{2}}\right)^{1 / \alpha} .
$$

[40] The integral range $A_{3}$ for porosity was approximated by computing the variance $D^{2}(V)$ for the recorded porosities
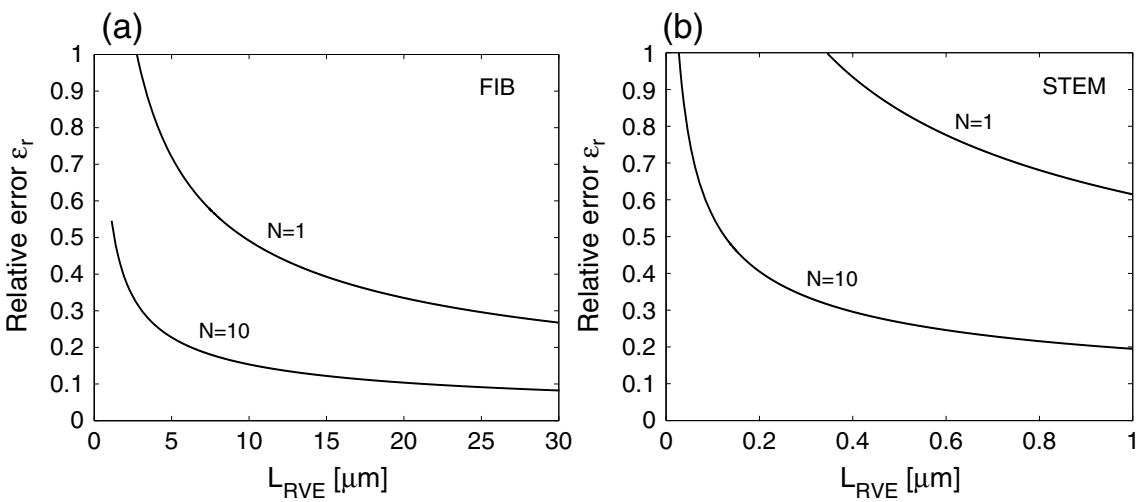

Figure 12. Relative precision $\varepsilon_{r}$ for $N=1$ and $N=10$ versus $L_{\mathrm{RVE}}$ of porosity calculated on base of (a) FIB-nt and (b) STEM tomography when applied to sample BDR (shaley facies). The plots give an idea of the representativity of different tomographic methods which were used to study Opalinus clay. 
a)

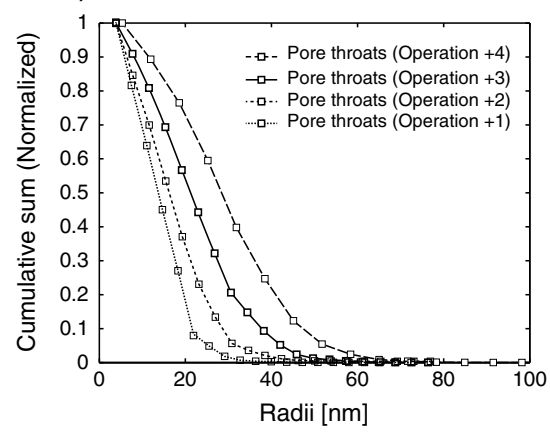

b)

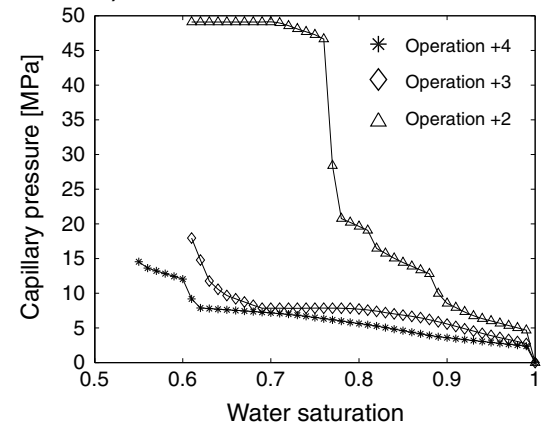

c)

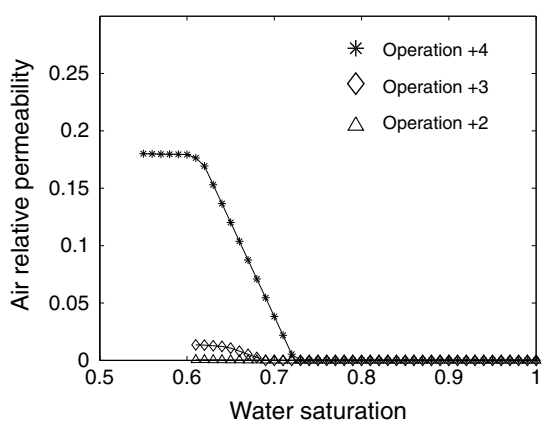

Figure 13. Prediction of gas transport properties for different pore microstructures (see Table 3). (a) Distributions of pore throat radii corresponding to different pore networks that were extracted from inflated pore structures (see Table 3). (b) Predicted capillary pressure curves and (c) relative permeabilities for different pore microstructures.

of the respective measuring cells of size $V$. Then, the integral range $A_{3}$ was obtained by fitting equation (9) to our data (Figures 10d, 10e, and 10f). Here one has the problem that the true mean value $M$ of porosity or clay content cannot be predicted from tomographic methods because they have been applied to volumes, of which sizes are far from infinite. The latter statement assumes that the true porosity can be obtained from a clay rock body of infinite size. In addition, each method has its own true value of porosity or clay content in dependence of the resolution limitation of the respective method. Thus, the calculated RVE is not only related to an error and number of realizations $N$ but also to the respective microstructural level that can be resolved by the different imaging methods. Here we assumed that bulk volume fractions of porosity and clay content that were determined by the different methods are valid estimates of the true volume fractions. This is supported by the fact that calculated mean values do not vary much in dependence of $L$ (Figure 10). In the case of porosity, this assumption is also supported by nitrogen adsorption analyses, which were applied to much larger volumes than FIB-nt and STEM, and yielded good agreement with tomographic methods for the respective microstructural level [Keller et al., 2011]. The values of $A_{3}, \alpha$, and volume fractions for each method are given in Table 4. Then, the size of the respective RVE for each method can be calculated for a given precision of the mean value that results from different realizations $N$ (i.e., independent measure cells with the size of the RVE). For $N=1$, Figure 11 shows calculated $L_{\mathrm{RVE}}\left(=V\left(1, \varepsilon_{r}\right)^{1 / 3}\right)$ as a function of relative precisions $\varepsilon_{r}$. Concerning the porosity in the clay matrix, the calculations yielded similar values of RVE for different samples and methods. For example, if we tolerate an $\varepsilon_{r}$ of $10 \%$, the RVE of porosity is in the $100 \mu \mathrm{m}$ range (Figure 11). Conformity between different samples and methods suggests that the RVE of porosity really exists, which of course cannot be assumed a priori. In the case of FIB-nt and STEM, it turns out that the expected relative error of a single realization (i.e., measurement) is $>10 \%$ because the edge length of the volume that can be analyzed is $<100 \mu \mathrm{m}$.

[41] Regarding the error related to FIB-nt and STEM, Figure 12 shows the calculated curves of $\varepsilon_{r}(L)$ for $N=1$ and $N=10$. The edge length of the cube that is analyzed by FIB-nt is about $10 \mu \mathrm{m}$. For this length, $\varepsilon_{r}$ ranges between
$40 \%-50 \%$ and $10 \%-20 \%$ for a single realization and ten realizations, respectively (Figure 12a). The figure also reveals that an increase of the size of the analyzed volume leads only to a minor reduction of error. If one is able to perform a single STEM realization with a sample size on the range of $400 \mathrm{~nm}$, the expected relative error is about $100 \%$, but for an effort of 10 realizations, the relative error will decease to about $30 \%$ (Figure $12 b$ ).

\section{Discussion}

[42] Continuum models are used to predict the behavior of radioactive waste deposits at various scales. Such methods assume that the host rock exists as a continuum, which in the case of porosity means that it is homogenously distributed over a certain length scale. More specifically, the continuum assumption depends on the idea of an RVE. Here we show that the validity of the continuum assumption can be verified by statistically analyzing pore space reconstructions based on tomographic data. Local porosity theory reveals that the porosity in the clay matrix possesses spatial homogeneity on the scale of a few microns (Figure 4). However, based on a statistical approach of RVE, it is shown that on this length scale, the relative error associated to the mean value of porosity is $>10 \%$ (Figure 12). If we accept a relative error of $10 \%$, the RVE of porosity is on the hundreds of micron scale (Figure 11). This length scale is larger than the one that can be analyzed by FIB-nt and STEM. This means that we either accept higher relative errors or that we perform more than one tomographic realization (Figure 12). The latter of which reduces the error to an acceptable value (Figure 12). In the case of STEM, the expected relative error for a single realization is far from being acceptable because the sample size is way too small (i.e., $0.1 \mu^{3}$ ) (Figure 12). Hence, if one wants to obtain statistically relevant results for Opalinus clay by means of STEM, then it is an inevitable condition to collect more than one 3-D analysis.

[43] Regarding pores with radii $>\sim 10 \mathrm{~nm}$, we found a percolation anisotropy related to the bedding in the sediments. Percolation threshold $\phi_{c}$ parallel to bedding is as low as $\sim 0.04$ porosity in the clay-rich matrix of the shaley facies and a little higher in the clay matrix of the sandy facies. The measured fraction of larger pores (i.e., radii $>\sim 10 \mathrm{~nm}$ ) is close or even higher than these values. Because larger pores control gas 
transport, this implies that gas percolation in the bedding plane (i.e., radii $>\sim 10 \mathrm{~nm}$ ) is reasonable in Opalinus clay. Gas percolation perpendicular to bedding, however, can be excluded because we found that this requires a volume fraction of larger pores higher than $\sim 0.12$, which could not be measured for Opalinus clay.

[44] In terms of gas transport, the calculated percolation threshold $\phi_{c}$ must be considered as "dry" percolation threshold because gas permeability depends on air filled porosity, which in turn depends on water saturation. Water is the wetting fluid, which is retained in angular corners due to capillary forces. In this way, it allows the simultaneous flow of the two phases. As a consequence, the critical volume fraction for air percolation in moist environment is smaller than the "dry" $\phi_{c}$. In addition, gas flow through an initially water-saturated pore space is controlled by capillary pressure effects. In Opalinus clay, full water saturation is indicated by the good agreement between porosities derived from densities and water content, respectively [Bossart and Thury, 2008]. Thus, replacement of water by a gas phase in Opalinus clay is largely controlled by the pore throat distribution, which in turn controls the pressure required to force gas into the largest pores. However, if the larger pores are unconnected and porosity is below the percolation threshold $\phi_{c}$, the pore space is largely impermeable to gas. Because porosity of Opalinus clay is apparently close the critical porosity $\phi_{c}$, at least for percolation within the bedding plane, changes in gas transport properties in case $\phi$ approaches $\phi_{c}$ from above might be of interest. In order to obtain an idea of these changes, we used the network simulator that was developed by Valvatne and Blunt [2004]. In order to use this simulator, an equivalent pore network was extracted on the basis of the reconstructed voxel representation of the pore microstructure by using an implementation of the maximum ball algorithm developed and written by Dong and Blunt [2009]. The result of this computation is a line skeleton with the topology or connectivity of the porous media. The network is subdivided into pore bodies (i.e., nodes) and pore throats (i.e., edges) with assigned properties (inscribed radius, volume, etc.) extracted from the original voxel representation. This allows predicting the transport properties (e.g., absolute permeability) of the microstructure. It is assumed that capillary forces will retain the wetting fluid in angular corners, which allows the simultaneous flow of two fluids along the same pore. Networks with only circular tubes are unable contain more than one fluid. Thus, network elements are square or triangular shaped, whereby the irregularities of the pore space is considered when defining the cross section of the angular network elements. A highly irregular pore shape is, for example, considered by an equivalent shaped triangle that has sharp corners where water remains in the corners and the gas phase occupies the center of the pore. Hence, the calculated capillary pressure is a direct function of the pore shape. Regarding details concerning the calculation of gas flow properties by using pore-scale network modeling, the interested reader is referred to the textbook of Valvatne [2009].

[45] The inflated pore structure models with $\phi=0.044$, $0.067,0.092$, and 0.12 (Table 3) were used as input models for the network simulator. As shown above, the pore spaces of these models are increasingly connected and thus allow studying the behavior of gas transport properties near the percolation threshold. Apart from porosity and connectivity, the pore throat radii increase also above the percolation threshold (Figure 13a). Approaching the percolation threshold from above, the predicted absolute permeabilities parallel to bedding decrease from $6.7 \mathrm{E}-18$ to $4.2 \mathrm{E}-21\left(\mathrm{~m}^{2}\right)$ with a drop in values near the percolation threshold (Table 3 ). The predicted values are of a similar order of magnitude when compared to the values that were obtained by recent airflow experiments on Opalinus clay $\left(\sim 6.1 \mathrm{E}-19\left(\mathrm{~m}^{2}\right)\right)$ [Romero et al., 2013]. Figure 13b shows the predicted capillary pressure curves for water drainage. Gas pressures required to force gas into to the pore structure models do not differ much because the largest pores in the entry planes are of similar size (Table 3). On the contrary, gas pressures required for displacing pore water increase drastically when approaching the percolation threshold (Figure 13b). In addition, it is predicted that gas-relative permeabilities decrease by at least 2 orders of magnitude when approaching the percolation threshold (Figure 13c). Gas emergence pressures predicted for models with low porosity (i.e., $\phi<\sim 0.067$ ) and a high fraction of small pore radii (Table 3 and Figure 13b, distribution labeled operation +2$)$ are much higher $(>\sim 50 \mathrm{MPa})$ than the one obtained by airflow experiments $(<\sim 20 \mathrm{MPa})$ [Romero et al., 2013; Marschall et al., 2005]. All these predictions suggest that gas flow is indeed controlled by pore structures characterized by a high fraction of pores with throat radii $>20 \mathrm{~nm}$. In our case, reasonable capillary pressures for displacing pore water are predicted if the fraction of pores with throat radii $20 \mathrm{~nm}$ exceeds 0.5 (Table 3 and Figure 13a, distributions labeled operation +3 and +4$)$.

[46] Pores in the fine-grained clay matrix of grain-supported microstructures (i.e., in the sandy facies of Opalinus clay) are better connected when compared to its counterpart from the shaley facies (Figure 6). Finite scaling reveals that the former pore microstructure may be characteristic for shale microstructure at the onset of percolation because percolation threshold (i.e., at least in $z$ direction) determined on the basis of one used scaling scheme exceeds bulk porosity of the sample (Figure 8 and Table 1). Since the pore space in the carbonates is very poorly connected (Figure 6), the influence of nonclayey layers on pore connectivity and percolation can be described as follows. The presence of a grain-supported framework suggests lower compaction of clay platelets, which may lead to higher porosity and connectivity of the clay matrix in the sandy facies. However, this trend may be inverted when the clay matrix itself falls below the percolation threshold (e.g., in carbonate-rich zones, where the clay matrix is reduced to isolated lenses). If indeed the porosity in the clay matrix is higher in grain-supported microstructures, layers with comparably low clay contents should have a higher permeability. Thus, spatial distribution of clay content in the host rock is a critical parameter and should be known as accurately as possible in order to evaluate the behavior of the waste repository.

[47] Finally, we would like to stress that total intergranular porosity (i.e., incl. pores with radii $\sim<10 \mathrm{~nm}$ ) in Opalinus clay is $>\sim 0.12$, which in combination with the calculated bulk percolation threshold suggests full connectivity of pore space in Opalinus clay. The role of this porosity for gas transport is difficult to be estimated, but it is certainly of high importance for diffusion in the liquid phase (e.g., adsorbed water).

\section{Conclusions}

[48] On the level of detail that can be resolved by FIB-nt (with a typical resolution of 10-20 nm) and for typical sample 
volume sizes with edge lengths of about $10 \mu \mathrm{m}$, the pore space in Opalinus clay possesses a spatial homogeneity. Percolation probability analysis and finite scaling reveals anisotropies in connectivity and percolation threshold. We found a higher connectivity and lower percolation threshold parallel to bedding when compared to those perpendicular to the bedding. It can also be suggested that connectivity between pores with radii $>\sim 10 \mathrm{~nm}$ changes significantly in the 0.04 0.07 porosity range. The pore structure that is resolved by FIB-nt corresponds to a porosity of around $0.02-0.07$ and can thus be interpreted as the pore microstructure at the onset of percolation. More geometrical details can be added by increasing the resolution of observation. FIB tomography can be performed down to $5 \mathrm{~nm}$ resolution, or alternatively STEM can be used. However, unfortunately, the improvement of resolution is usually associated with a decrease in sample size. The analyses show that a single STEM tomography realization allows no final conclusion on the homogeneity behavior of porosity on the level of detail that can be resolved by STEM, which is due to the small sample size. Based on the results from FIB tomography, it must be assumed that transport in Opalinus clay occurs predominately within the porous clay matrix. These rocks are thus permeable for gas when the clay matrix itself forms a percolating network on a larger scale.

[49] Focus was also spent on the calculation of the RVE related to different levels of resolution. With increasing resolution, the sample size that can be analyzed decreases along with the captured geometric features. As a consequence, more realizations are necessary when using high-resolution methods such as STEM in order to capture enough of the geometric content so that method provides representative information on the microstructure.

[50] Regarding gas transport on the millimeter to centimeter scale, one might argue that transport occurs within nonclayey layer materials. In the case of carbonate layers, the pores are larger when compared to the pores in the clay matrix, which renders them as a potential pathway for gas transport. However, porosity in carbonates is only in the 0.03 range, and the pore space is unconnected. Thus, at least for the analyzed sample from the sandy facies of Opalinus clay, gas transport in carbonate layers is doubtful. Nevertheless, nonclayey material might still have an important influence on gas transport properties but for a different reason. In a grain-supported shale microstructure, the clay matrix between nonclayey grains and layers is characterized by a higher porosity and better connectivity when compared to matrix-supported microstructures of clayrich shales. This is likely due to the lower degree of compaction of clay platelets in grain-supported microstructures.

[51] Acknowledgments. This work was funded by the Swiss National Cooperative for the Disposal of Radioactive Waste (NAGRA) as part of the SHARC consortium, a research collaboration between the Commonwealth Scientific and Industrial Research Organization (CSIRO), Curtin University of Technology, and NAGRA.

\section{References}

Andra (2005), Dossier 2005 Argile_ evaluation de la faisabilité du stockage géologique en formation argileuse profonde-Rapport de synthèse, Andra, Chatenay-Malabry, Cedex, France. [Available at: http://www.Andra.fr].

Bear, J. (1993), Modeling flow and contaminant transport in fractured rocks, in Flow and Contaminant Transport in Fractured Rocks, edited by J. Bear, C. Tsang, and M. Ghislain, pp.1-37, Academic, New York.

Biswal, B., C. Manwart, and R. Hilfer (1998), Three-dimensional local porosity analysis of porous media, Physica A, 255, 221-241.
Bossart, P., and M. Thury (2008), Mont Terri Rock Laboratory: Project, programme 1996-2007 and results, Rep. 3, Swiss Geol. Surv., Wabern.

Bunde, A., and S. Havlin (1995), Percolation I, in Fractals and Disordered Systems, edited by A. Bunde and S. Havlin, pp. 59-114, Springer, Berlin. Clayton, C. J., and S. J. Hay (1994), Gas migration from accumulation to surface, Bull. Geol. Soc. Den., 41, 12-23.

Cosenza, P., C. Camerlynck, M. Fleury, Y. Géraud, T. Kremer, P. Landrein, P. Lebon, D. Prêt, F. Villieras, and M. Zamora (2012), Petrophysical characterization of Callovo-Oxfodian argillites from the deep borehole Andra EST433 of Montiers-sur-Saulx (Meuse, France), in Clays in Natural and Engineered Barriers for Radioactive Waste Confinement, 4th International Meeting, Montpellier, October, pp. 143-144, Andra, Chatenay-Malabry, Cedex, France.

Dong, H., and M. J. Blunt (2009), Pore-network extraction from microcomputerized-tomography images, Phys. Rev., E80, 036307.

Garboczi, E. J., K. A. Snyder, J. F. Douglas, and M. F. Thorpe (1995), Geometrical percolation threshold of overlapping ellipsoids, Phys. Rev., 52, 819-828.

Hilfer, R. (1991), Geometric and dielectric characterization of porous media, Phys. Rev., 44, 60-75.

Hilfer, R., and R. Helmig (2004), Dimensional analysis and upscaling of two phase flow in porous media with piecewise constant heterogeneities, $A d v$. Water Resour., 27, 1033-1040.

Horseman, S. T., J. J. W. Higgo, J. Alexander, and J. F. Harrington (1996), Water, gas and solute movement through argillaceous media. Report for the NEA working group on a measurement and physical understanding of groundwater flow through argillaceous media ("clay club"), a sub-group of the NEA coordinating group in site evaluation and design of experiments for radioactive waste disposal (SEDE), NEA/OECD Rep, CC-96/1, Organ. for Econ. Co-op. and Dev., Paris.

$\mathrm{Hu}, \mathrm{J}$, and P. Stroeven (2005), Local analysis of pore structure in cement paste, Cem. Concr. Res., 35, 233-242.

Hunt, A., and R. Ewing (2009), Percolation Theory for Flow in Porous Media, Lect. Notes in Phys., vol. 771, Springer, Berlin.

Kanit, T., S. Forest, I. Gailliet, V. Mounoury, and D. Jeulin (2003), Determination of the representative volume for random composites: Statistical and numerical approach, Int. J. Solids Struct., 40, 3647-3679.

Keller, L. M., L. Holzer, R. Wepf, and P. Gasser (2011), 3D Geometry and topology of pore pathways in Opalinus clay: Implications for mass transport, Appl. Clay Sci., 52, 85-95.

Keller, L. M., P. Schuetz, R. Erni, F. Lucas, P. Gasser, and L. Holzer (2013), Characterization of multi-scale microstructural features in Opalinus clay, Microporous Mesoporous Mater., 179, 83-94, doi:10.1016/j. micromeso.2012.11.029.

Lantuejoul, C. (1991), Ergodicity and integral range, J. Microsc., 161, 387-403.

Liu, J., and K. Regenauer-Lieb (2011), Application of percolation theory to microtomography of structured media: Percolation threshold, critical exponents, and upscaling, Phys. Rev. E, 83, 016106-1-13.

Marschall, P., S. Horseman, and T. Gimmi (2005), Characterisation of gas transport properties of the Opalinus clay, potential host rock formation for radioactive waste disposal, Oil Gas Sci. Technol., 60, 121-139.

Matheron, G. (1971), The Theory of Regionalized Variables and Its Application, Paris School of Mines publications, No. 5, $211 \mathrm{pp}$.

Münch, B., and L. Holzer (2008), Contradicting geometrical concepts in pore size analyzes attained with electron microscopy and mercury intrusion, J. Am. Ceram. Soc., 91, 4059-4067.

Nagra (2002), Projekt Opalinuston-Synthese der geowissenschafftlichen Untersuchungsergebnisse. Entsorgungsnachweis für abgebrannte Brennelemente, verglaste hochaktive sowie langlebige mittelaktive Abfälle, Nagra Tech. Rep. NTB, 02-03, Nagra Weinfelden, Switzerland.

Nagra (2004), Effects of post-disposal gas generation in a repository for spent fuel, high-level waste and long-lives intermediate waste sited in the Opalinus clay, Nagra Technical report NTB, 04-06, Wettingen, Switzerland.

Pringle, D. J., J. E. Miner, H. Eicken, and K. M. Golden (2009), Pore-space percolation in sea ice single crystals, J. Geophys. Res., 114, C12017, doi:10.1029/2008JC005145.

Robinet, J.-C., P. Sardini, D. Coelho, J. C. Parneix, D. Prêt, E. Boller, and S. Altmann (2012), Effects of mineral distribution at mesoscopic scale on solute diffusion in a clay-rich rock: Example of the CallovoOxfordian mudstone Bure, France, Water Resour. Res., 48, W05554, doi:10.1029/2011WR011352.

Romero, E., R. Senger, P. Marschall, and R. Gomez (2013), Air tests on lowpermeability claystone formations. Experimental results and simulations, in Multiphysical Testing of Soils and Shales, edited by L. Laloui and A. Ferrari, pp. 69-83, Springer, Berlin.

Stauffer, D., and A. Aharony (1995), Percolation Theory: An Introduction, $\mathrm{VCH}$, Weinheim, Germany.

Valvatne, P. (2009), Predictive Pore-Scale Modelling of Multiphase Flow, VDM Verlag, Saarbrucken, Germany.

Valvatne, P. H., and M. J. Blunt (2004), Predictive pore-scale modeling of two-phase flow in mixed wet media, Water Resour. Res., 40, W07406, doi:10.1029/2003WR002627. 